\title{
Skin Segmentation Using YUV and RGB Color Spaces
}

\author{
Zaher Hamid Al-Tairi ${ }^{*}$, Rahmita Wirza Rahmat*, M. Iqbal Saripan*, and \\ Puteri Suhaiza Sulaiman*
}

\begin{abstract}
Skin detection is used in many applications, such as face recognition, hand tracking, and human-computer interaction. There are many skin color detection algorithms that are used to extract human skin color regions that are based on the thresholding technique since it is simple and fast for computation. The efficiency of each color space depends on its robustness to the change in lighting and the ability to distinguish skin color pixels in images that have a complex background. For more accurate skin detection, we are proposing a new threshold based on RGB and YUV color spaces. The proposed approach starts by converting the RGB color space to the YUV color model. Then it separates the $Y$ channel, which represents the intensity of the color model from the $\mathrm{U}$ and $\mathrm{V}$ channels to eliminate the effects of luminance. After that the threshold values are selected based on the testing of the boundary of skin colors with the help of the color histogram. Finally, the threshold was applied to the input image to extract skin parts. The detected skin regions were quantitatively compared to the actual skin parts in the input images to measure the accuracy and to compare the results of our threshold to the results of other's thresholds to prove the efficiency of our approach. The results of the experiment show that the proposed threshold is more robust in terms of dealing with the complex background and light conditions than others.
\end{abstract}

Keywords-Skin Segmentation, Thresholding Technique, Skin Detection, Color Space

\section{INTRODUCTION}

Skin detection is an interesting and challenging topic for many researchers as it is used in many applications such as, hand and face tracking, sign language recognition, human-computer interaction, and others. The difficulty of skin detection is due to the variation of people's race where each race has a different skin tone from the others. The difference in people's skin is not the only problem as there other factors such as the light conditions where the variation in luminance limits the accuracy of skin segmentation.

There are many skin color spaces like RGB, HSV, YCbCr, YIQ, YUV, etc. that are used for skin color segmentation. The RGB color model represents the colors that are in the red, green, and blue planes and does not separate the luminance from the chrominance components, which makes it a poor choice for color analysis and color based recognition [1]. The conversion from RGB color space to the HSV color model is time consuming due to the time it takes to do a nonlinear transmission [2]. To overcome the previous problems, we have proposed a new threshold,

\footnotetext{
Manuscript received April 17, 2013; first revision September 24, 2013; accepted November 28, 2013.

Corresponding Author: Zaher Hamid Al-Tairi (zaher_tu@yahoo.com)

* University Putra Malaysia (UPM), Selangor, Malaysia (\{zaher_tu, zaher78hamid, breave78heart, husamzah\}@yahoo.com)
} 
which is based on the YUV and RGB color spaces. Our approach is able to benefit from the characteristics of each color model for enhancing the accuracy of skin detection.

\section{RELATED WORK}

There are many techniques for skin color segmentation, such as the Gaussian Mixture Model, color histogram, and thresholding. Some of the skin detection methods that adopt the threshold manner will be discussed in this paper. Sobottka and Pitas [3] presented a method for skin color segmentation based on HSV color space using fixed threshold values to extract the face region. Due to the variation in light conditions, they adopted shape features to enhance the face detection feature. Jusoh et al. [4] introduced an approach for skin color detection, which is based on HSV and RGB color spaces, to improve the segmentation process by using two thresholds. The first threshold is applied to the hue channel, and the second threshold is applied to the RGB model. Another method that combines two color models ( $\mathrm{HSV}$ and $\mathrm{YCgCr}$ ) was proposed by Ghazali et al. [5] for extracting the face region. It is based on thresholding techniques. Segmentation by combining more than one color model improves the accuracy of skin detection. Ghotkar and Kharate [6] described a hand segmentation method by using a threshold technique for hand gesture recognition. They made a comparison between three color spaces (HSV, HSL, and HTS) and found that the last one gave better results than the others. Jagadesh et al. [7] presented an approach for skin segmentation using the bivariate Pearsonian Type-IIb Mixture Model. They used the hue and saturation components of HSV color space to distinguish the skin and non-skin pixels, which they based on the threshold values and the Likelihood method, to enhance the accuracy of the results. However, using HSV color space in skin segmentation is time consuming due to the time it takes for non-linear transformation to occur between the RGB and HSV color models.

Chai and Ngan [8] suggested a method for extracting the face region from an input image using the thresholding technique. The color space used in this approach is the $\mathrm{YCrCb}$ color model. It was used due to its efficiency for modeling skin color and for its use in video coding. Another method that uses $\mathrm{YCbCr}$ color space with fixed thresholding to extract the face region is described by Marius et al. [9]. It applies morphological operations to reduce the unwanted regions. However, skin detection using the $\mathrm{YCbCr}$ color model in the previous methods is unsuitable for some races, such as black people.

Kukharev and Nowosielski [2] extracted the skin regions by using the RGB and $\mathrm{YCbCr}$ color spaces to improve the accuracy of the skin detection. For fast face segmentation de Dios and Garcia [10] proposed a YCgCr color model with two thresholds. Although they enhanced the face detection by rotating the $\mathrm{Cr}$ and $\mathrm{Cg}$ axis in the chrominance channel, the process of conducting segmentationat a higher level of accuracy still needs to be improved. Almohair et al. [11] introduced a method to distinguish the human skin from the color images that contain luminance. They did so by using the thresholding technique, which is considered to be an effective tool for enhancing the segmentation process. A new color model for skin segmentation that is based on RGB color space has been introduced to speed up the process of classifying the pixels by converting the $3 \mathrm{D}$ space of RGB to $1 \mathrm{D}$. This method uses the thresholding technique to distinguish skin pixels from the non-skin pixels. Although the segmentation is fast, the results need to be enhanced due to the absence of the red channel [1]. A Fuzzy YCbCr color space and 
threshold technique was described by Iraji and Yavari [12] to deal with the lighting effects and to enhance the accuracy of the skin color segmentation. Prema and Manimegalai [13] presented a hybrid approach to extract the skin regions in an image with a complex background by using a fixed threshold.

The previous discussion presents the idea that using more than one color space with a threshold is considered to be an effective tool for skin color segmentation. In this paper, a new skin segmentation method, which adopts a threshold technique that is based on the YUV and RGB color models to improve the accuracy of skin detection, is introduced. To prove the effectiveness of this method, we compared it with the methods in $[2,3,14]$. The results show that the proposed method detects more skin regions than the other methods do.

\section{COLOR MOdeLS}

Many different types of color models are used for skin detection. Each one differs from the others in terms of the manner of transformation (linear or non-linear), the robustness to adapt to light changing, and shadow noises. The paragraphs below are a short description for the color models that were used in this experiment.

\subsection{RGB Color Model}

It consists of the three main colors: red, green, and blue. Combining the luma and chromatic components in this color space make it more sensitive to noise than others. The results of the proposed threshold were compared to the results of the RGB color space, which was based on the following threshold values described by [14]:

$$
\left\{\begin{array}{c}
\mathrm{R}>95 \& G>40 \& B>20 \\
\max (\mathrm{R}, \mathrm{G}, \mathrm{B})-\min (\mathrm{R}, \mathrm{G}, \mathrm{B})>15 \\
|\mathrm{R}-\mathrm{G}|>15 \& R>G \& R>B
\end{array}\right.
$$

\subsection{HSV Color Model}

This color model is represented by hue, saturation, and value. The hue channel defines the color itself, whereas, the saturation channel describes the light combined with hue, and the value determines the image brightness. The conversion from RGB to HSV is based on the following equations mentioned by [15]:

$$
H=\left\{\begin{array}{c}
\mathrm{Hi} \quad \mathrm{B} \leq \mathrm{G} \\
360-\mathrm{Hi} \quad \mathrm{B}>G
\end{array}\right.
$$

Where:

$$
\begin{gathered}
\mathrm{Hi}=\arccos \left(\frac{\frac{1}{2\{(\mathrm{R}-\mathrm{G})+(\mathrm{R}-\mathrm{B})\}}}{\sqrt{(\mathrm{R}-\mathrm{G}) \uparrow 2+(\mathrm{R}-\mathrm{G})(\mathrm{G}-\mathrm{B})}}\right) \\
\mathrm{S}=\frac{\max (\mathrm{R}, \mathrm{G}, \mathrm{B})-\min (\mathrm{R}, \mathrm{G}, \mathrm{B})}{\max (\mathrm{R}, \mathrm{G}, \mathrm{B})}
\end{gathered}
$$




$$
\mathrm{V}=\frac{\max (\mathrm{R}, \mathrm{G}, \mathrm{B})}{255}
$$

The threshold values used to distinguish the skin regions, as proposed by Sobottka and Pitas [3], are as follows:

$$
\left\{\begin{array}{c}
0.23<S<0.68 \\
0<H<50
\end{array}\right.
$$

\subsection{YCbCr Color Model}

The $\mathrm{YCbCr}$ color space consists of the $\mathrm{Y}$ channel, which represents the luminance component, and the $\mathrm{Cb}$ and $\mathrm{Cr}$ channels, which describe the chrominance components. The separation of luma from chromatic makes this color model interesting for skin color detection. The transformation from RGB to $\mathrm{YCbCr}$ is simple and is defined as follows [15]:

$$
\begin{gathered}
\mathrm{Y}=0.299 \mathrm{R}+0.587 \mathrm{G}+0.114 \mathrm{~B} \\
\mathrm{Cb}=(\mathrm{B}-\mathrm{Y}) * 0.564+128 \\
\mathrm{Cr}=(\mathrm{R}-\mathrm{Y}) * 0.713+128
\end{gathered}
$$

The skin pixels can be extracted by using the threshold values, as follows [2]:

$$
\left\{\begin{array}{c}
135<C r<180 \\
85<C b<135 \\
\mathrm{Y}>80
\end{array}\right.
$$

\subsection{IHLS Color Model}

The Improved Hue, Luminance, and Saturation (IHLS) color model is introduced to reduce the effect of the lighting variations by removing the normalization of the saturation. The saturation of achromatic pixels is low and the luminance and saturation are independent of each other. The IHLS color model is generated from the RGB color model using the following equations [16]:

$$
\begin{array}{r}
\mathrm{Y}=0.2126 \mathrm{R}+0.7152 \mathrm{G}+0.0722 \mathrm{~B} \\
\mathrm{~S}=\frac{\max (\mathrm{R}, \mathrm{G}, \mathrm{B})-\min (\mathrm{R}, \mathrm{G}, \mathrm{B})}{255} \\
\mathrm{H}^{\prime}=\arccos \left(\frac{\mathrm{R}-\frac{\mathrm{G}}{2}-\frac{\mathrm{B}}{2}}{\left(\mathrm{R}^{2}+\mathrm{G}^{2}+\mathrm{B}^{2}-\mathrm{RG}-\mathrm{RB}-\mathrm{BG}\right)^{\frac{1}{2}}}\right) \\
\mathrm{H}=\left\{\begin{array}{c}
360^{\circ}-\mathrm{H}^{\prime} \quad \mathrm{B}>\mathrm{G} \\
\mathrm{H}^{\prime} \quad \text { otherwise }
\end{array}\right.
\end{array}
$$

The skin color regions are detected based on the following static thresholds [17]:

$$
\left\{\begin{array}{c}
0 \leq \mathrm{H} \leq 50 \\
0.1 \leq \mathrm{S} \leq 0.9
\end{array}\right.
$$




\subsection{YIQ Color Model}

The YIQ color space is used in Japanese and North American TVs to encode color images. It consists of three components: the Y component represents the luminance, whereas, the I and Q components represent the chromatic. The independence of the luminance component from chromatic components decreases the lighting effects. The transformation from the RGB color model to YIQ color model is given by the following formulations [18]:

$$
\left(\begin{array}{l}
Y \\
I \\
Q
\end{array}\right)=\left(\begin{array}{lll}
+0.299 & +0.587 & +0.114 \\
+0.596 & -0.275 & -0.322 \\
+0.212 & -0.523 & +0.311
\end{array}\right) \cdot\left(\begin{array}{l}
R \\
G \\
B
\end{array}\right)
$$

The following static filters that are described by [19] are used for skin color segmentation:

$$
\left\{\begin{array}{c}
44<Y<223 \\
0<I<64
\end{array}\right.
$$

\subsection{YIQ and HSV Color Model}

The combination of two color models for skin color segmentation is more robust than using a single color model. As such, skin detection is obtained based on the combination between YIQ and HSV color models using the following thresholds [20]:

$$
\left\{\begin{array}{c}
20 \leq \mathrm{I} \leq 90 \\
0.20<S<0.75 \\
\mathrm{~V}>0.35 \\
0<H<25
\end{array}\right.
$$

\subsection{YUV Color Model}

The YUV color model is used in some TV systems, such as NTSC (National Television System Committee) and PAL (Phase Alternation Line). The luminance channel (Y) is separated from the chromatic channels $(\mathrm{U}, \mathrm{V})$ to reduce the effect of lighting variations. The conversion of the RGB color model to YUV color model is achieved using the equations given below [21]:

$$
\left(\begin{array}{l}
Y \\
U \\
V
\end{array}\right)=\left(\begin{array}{lll}
+0.257 & +0.504 & +0.098 \\
-0.148 & -0.291 & +0.439 \\
+0.439 & -0.368 & -0.071
\end{array}\right) \cdot\left(\begin{array}{l}
R \\
G \\
B
\end{array}\right)+\left(\begin{array}{c}
16 \\
128 \\
128
\end{array}\right)
$$

The following equations represent the range of skin colors [23]:

$$
\left\{\begin{array}{l}
65<Y<170 \\
85<U<140 \\
85<V<160
\end{array}\right.
$$

\subsection{YIQ and YUV Color Models}

The YIQ and YUV color models are combined together for robust skin color detection. Listed below are the static filters that are described by [21] for skin regions extraction: 


$$
\left\{\begin{array}{c}
70<Y<175 \\
20<U<102 \\
-48<\theta<150
\end{array}\right.
$$

Where:

$$
\theta=\tan ^{-1}(|\mathrm{~V}| /|\mathrm{U}|)
$$

\subsection{RGB and YUV Color Models}

The RGB and YUV color models are combined together to overcome the control limits in these two color models for achieving the best skin color segmentation. The saturation parameter Ch in the YUV color model is used to reduce the noise and it's described as follows [22]:

$$
30 \leq \mathrm{Ch} \leq 220
$$

Where:

$$
\mathrm{Ch}=\sqrt{\mathrm{U}^{2}+\mathrm{V}^{2}}
$$

\subsection{YCgCr Color Model}

The $\mathrm{YCgCr}$ color model is a variant of the $\mathrm{YCbCr}$ color model in which the $\mathrm{Cg}$ component is used instead of the $\mathrm{Cb}$ component. The transformation from $\mathrm{RGB}$ to $\mathrm{YCgCr}$ is given below [23]:

$$
\left(\begin{array}{c}
\mathrm{Y} \\
\mathrm{Cg} \\
\mathrm{Cr}
\end{array}\right)=\left(\begin{array}{ccc}
+65.481 & +128.553 & +24.966 \\
-81.085 & +112 & -30.915 \\
+112 & -93.768 & -18.214
\end{array}\right) \cdot\left(\begin{array}{l}
\mathrm{r} \\
\mathrm{g} \\
\mathrm{b}
\end{array}\right)+\left(\begin{array}{c}
16 \\
128 \\
128
\end{array}\right)
$$

The following thresholds are introduced by [25] to extract the skin color regions:

$$
\left\{\begin{array}{c}
76 \leq \mathrm{Cg} \leq 125 \\
136 \leq \mathrm{Cr} \leq 202
\end{array}\right.
$$

\section{The Research Method}

The YUV color space was chosen due to the fast transformation of the RGB model. The Y channel represents the luminance of the color, while the $\mathrm{U}$ and $\mathrm{V}$ channels represent the chrominance. Separating the luma from the chromatic reduces the effect of light changing and shadow noises. This method starts by converting the RGB color space to the YUV color space using the following equations [18]:

$$
\left(\begin{array}{l}
Y \\
U \\
V
\end{array}\right)=\left(\begin{array}{lll}
+0.299 & +0.587 & +0.114 \\
-0.147 & -0.289 & +0.436 \\
+0.615 & -0.515 & -0.100
\end{array}\right) \cdot\left(\begin{array}{l}
\mathrm{R} \\
\mathrm{G} \\
\mathrm{B}
\end{array}\right)+\left(\begin{array}{c}
0 \\
128 \\
128
\end{array}\right)
$$

The skin detection threshold, which is based on the YUV color model, was built based on 200 faces with different colors. The threshold values used to detect human skin using $\mathrm{U}$ and $\mathrm{V}$ 
channels were determined by testing the boundary of skin colors with the help of the color histogram, as shown in Fig. 2 The threshold values, which are used to segment skin regions based on the RGB color space, were extracted by Kovac et al. [14] and were modified for our proposed method, as shown in Eq. (26).

$$
\left\{\begin{array}{c}
80<U<130 \\
136<V<200 \\
\mathrm{~V}>U \\
\mathrm{R}>80 \& G>30 \& B>15 \\
|\mathrm{R}-\mathrm{G}|>15
\end{array}\right.
$$

The following flowchart shows the process of skin color segmentation:

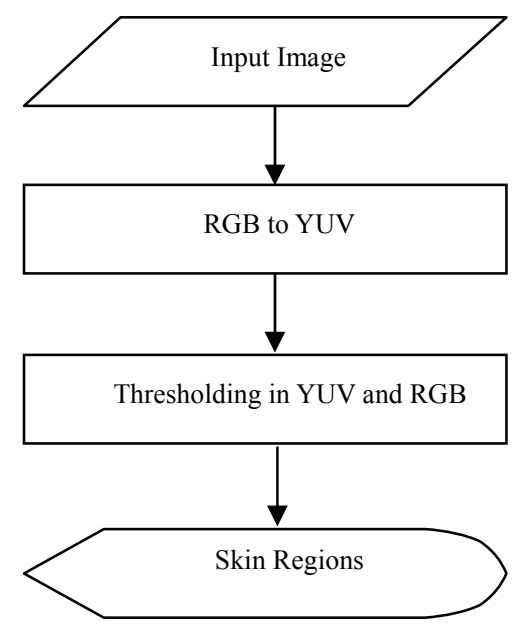

Fig. 1. Skin segmentation process

\section{RESULts}

The presented method was tested on 300 images selected from the Labeled Faces in the Wild Home database [24]. The size of each image is $250 \times 250$ and they vary in brightness and background. They also represent different races of people. A comparison was made between the introduced method and the previous methods in the color spaces of RGB, YCbCr, HSV, IHLS, YIQ, YUV, YIQ-HSV, YIQ-YUV, RGB-YUV, and YCgCr to prove the effectiveness of our suggested method. The experimental results show that the proposed method detected skin regions with low false positive and false negative rates and at a high detection rate. 


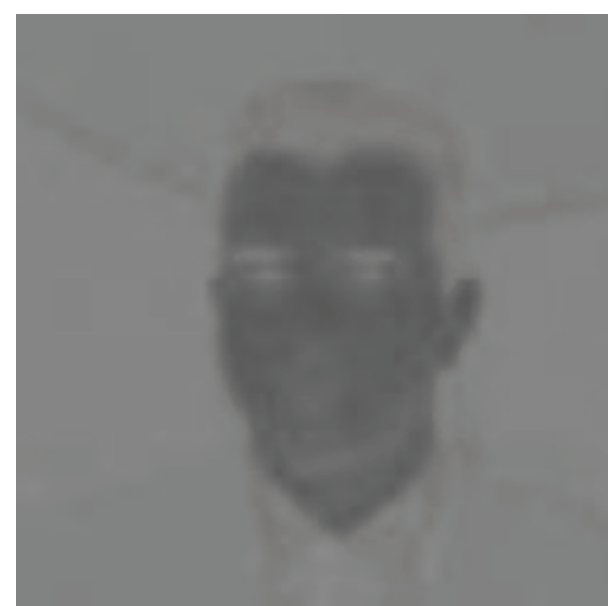

(a)

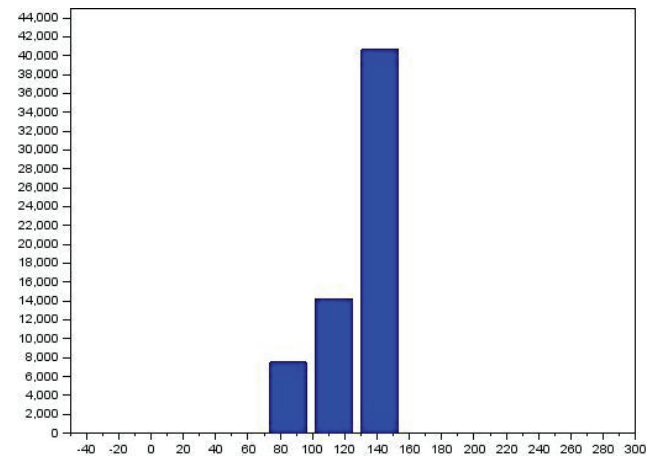

(c)

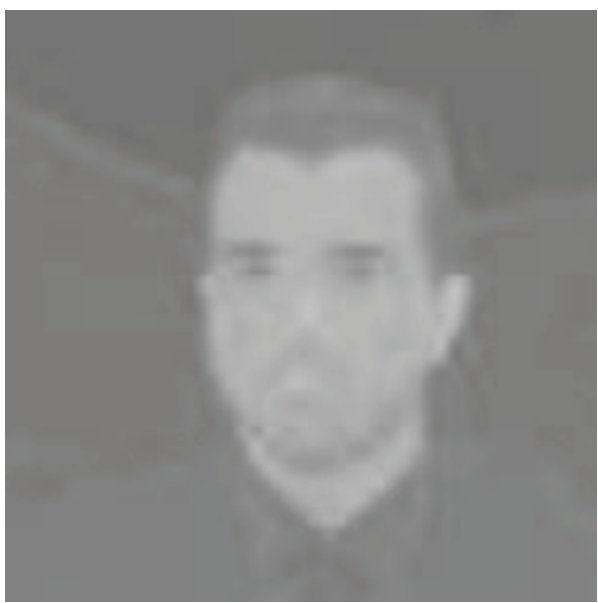

(b)

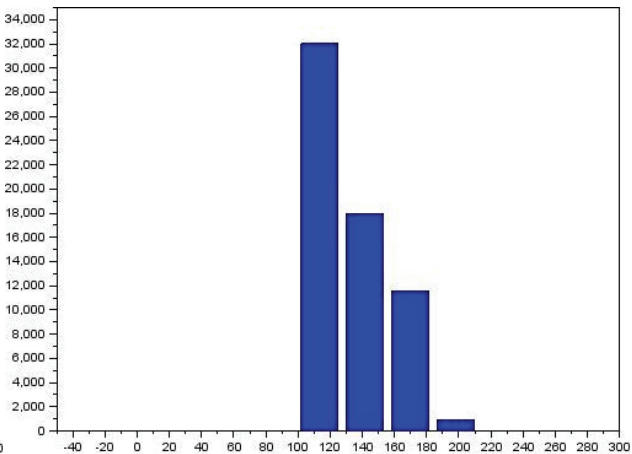

(d)

Fig. 2. Shows the $U$ and $V$ channels and their respective histograms: (a) $U$ channel, (b) $V$ channel, (c) histogram of the $U$ channel, and (d) histogram of the $V$ channel

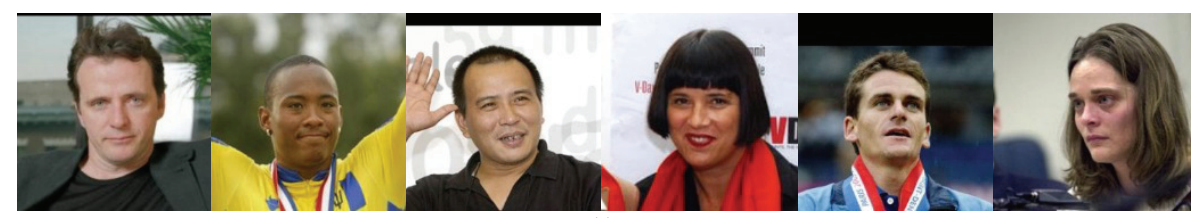

(a)

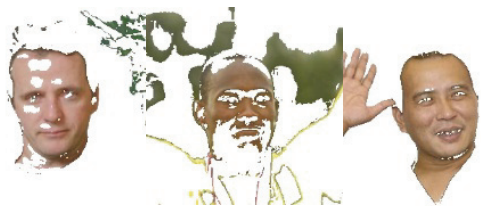

(b) 

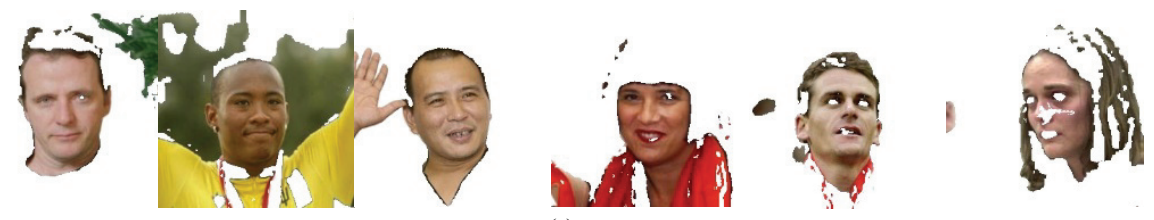

(c)
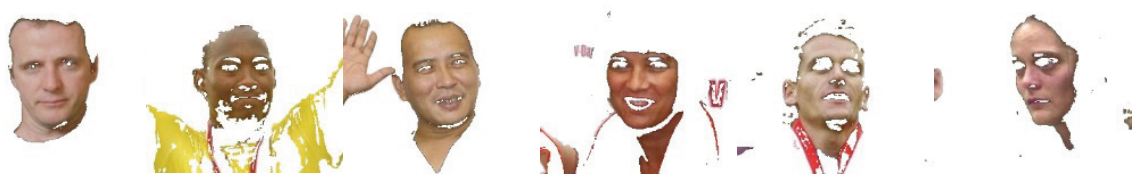

(d)
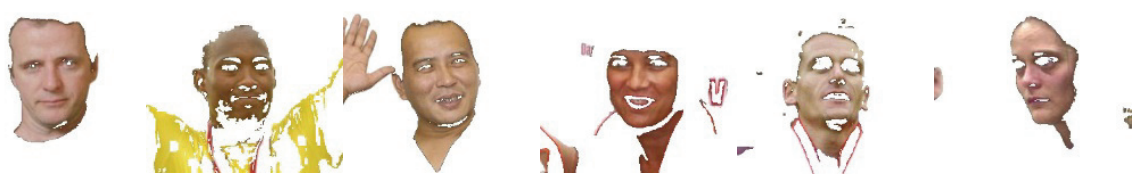

(e)
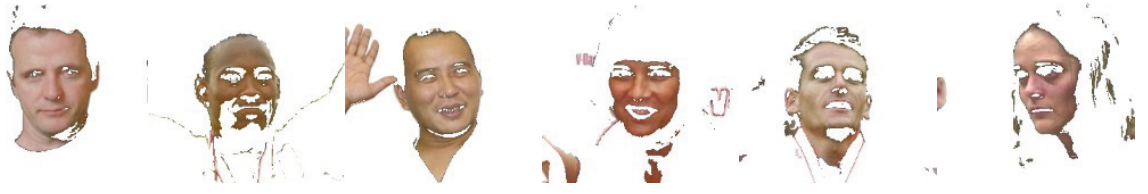

(f)
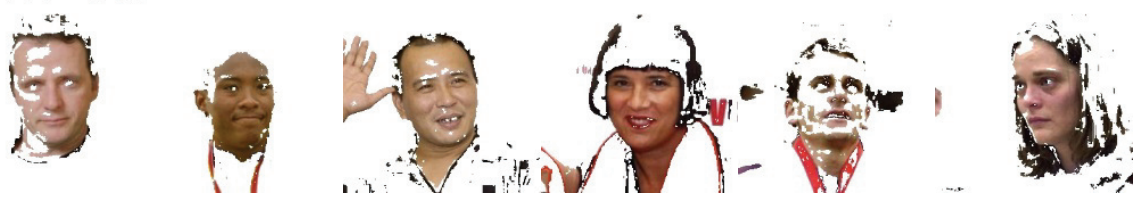

(g)
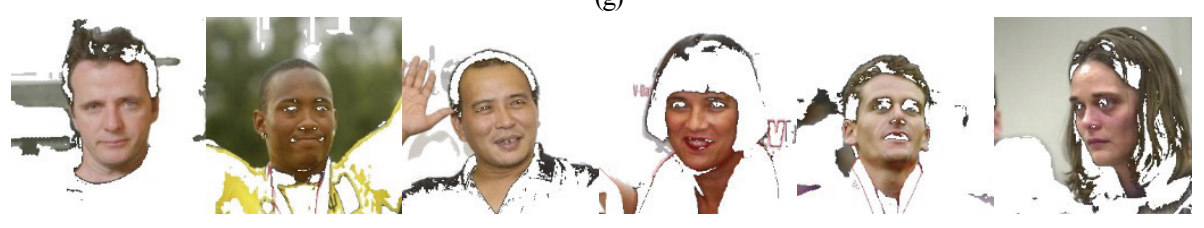

(h)
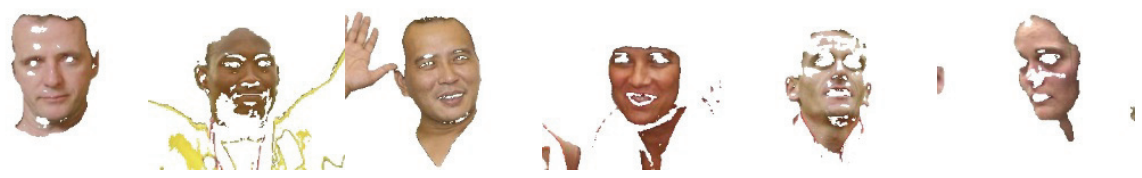

(i) 

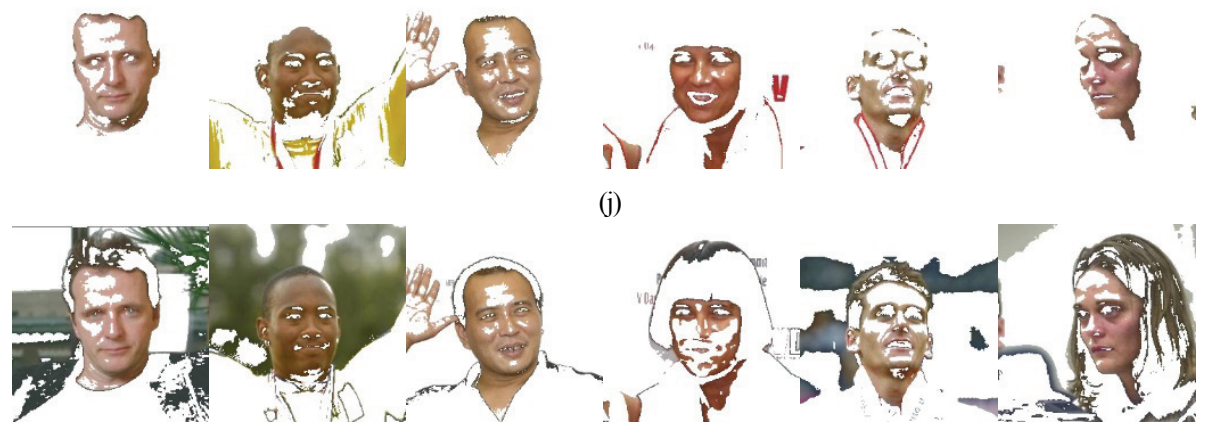

(j)

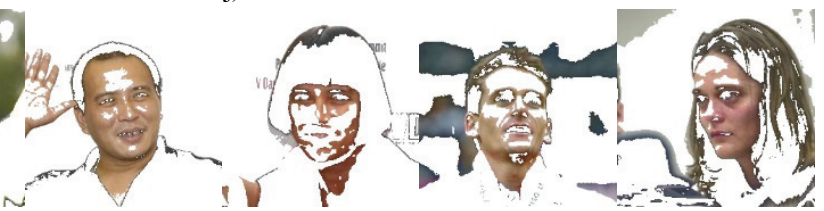

(k)
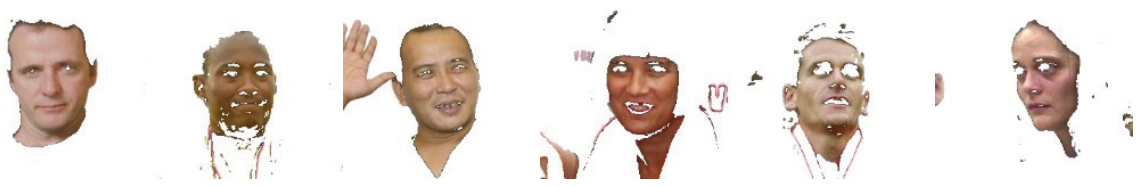

(1)

Fig. 3. Shows some of the results of the skin color segmentation process using different color models: (a) original images, (b) HSV, (c) IHLS, (d) RGB, (e) RGB\&YUV, (f) YCbCr, (g)

YCgCr, (h) YIQ, (i) YIQ\&HSV, (j) YIQ\&YUV, (k) YUV, and (l) YUV \& RGB (proposed method).

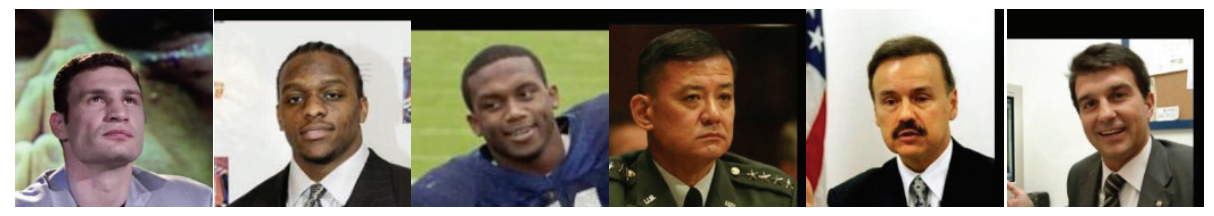

(a)
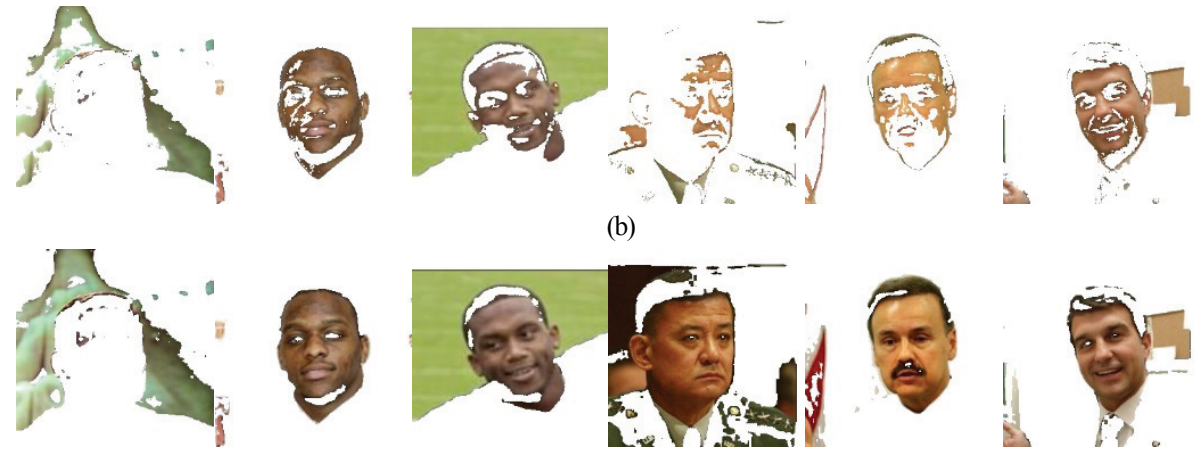

(c)
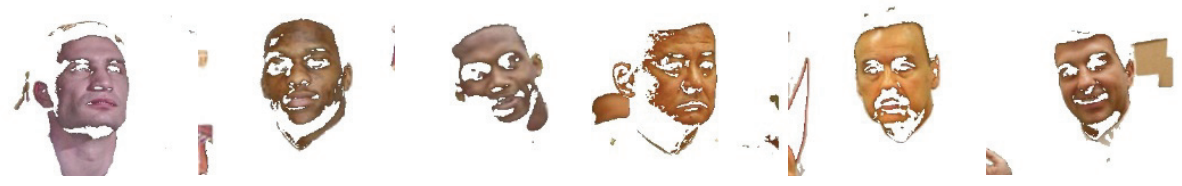

(d) 


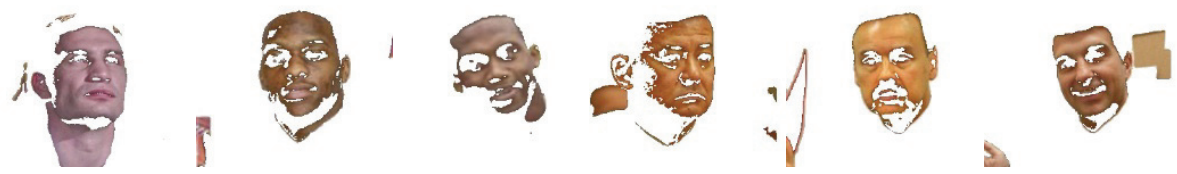

(\%)

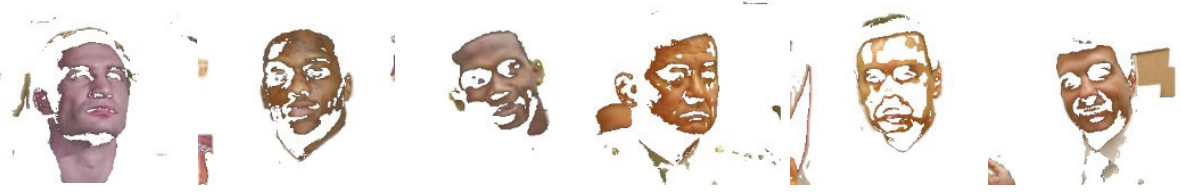

(f)
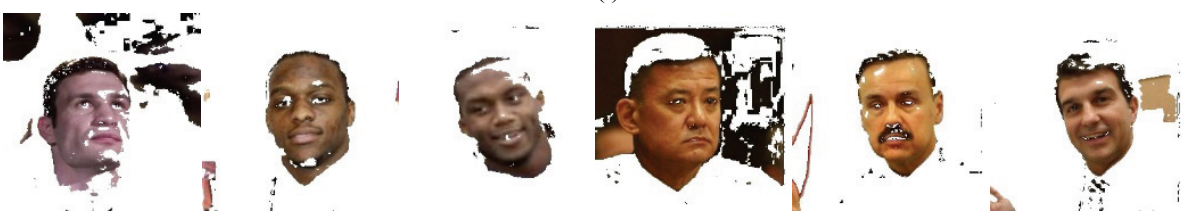

(g)
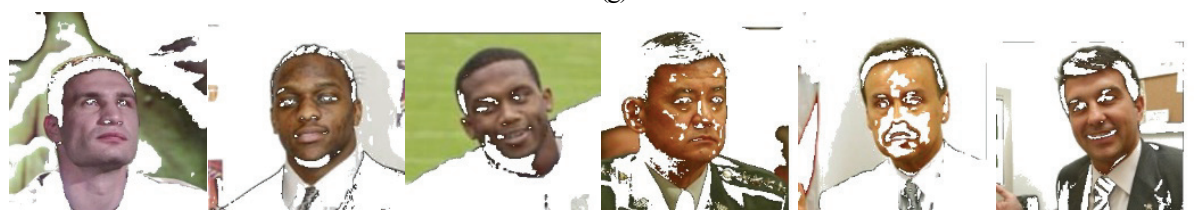

(h)

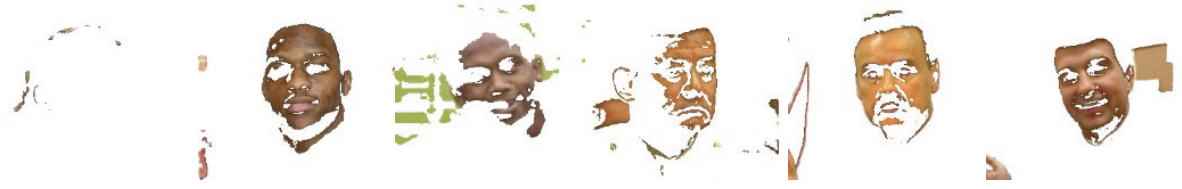

(i)

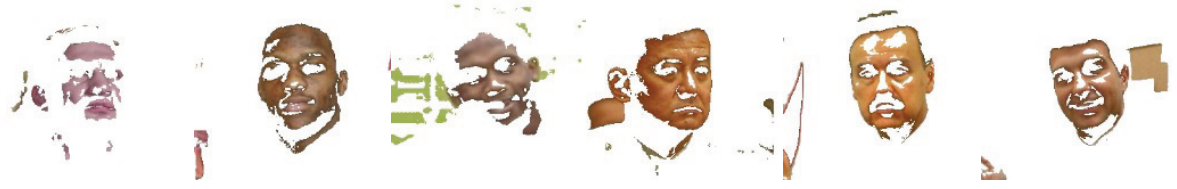

(j)

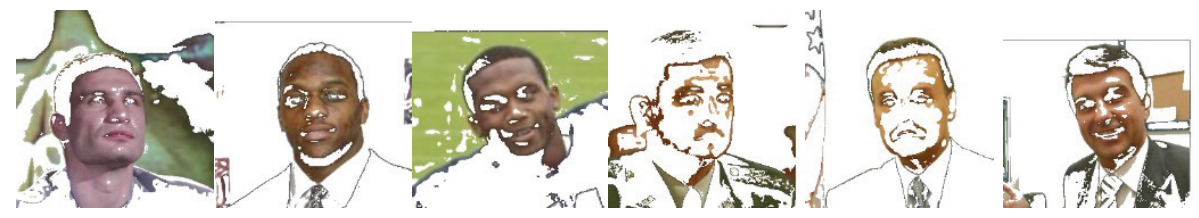

(k) 

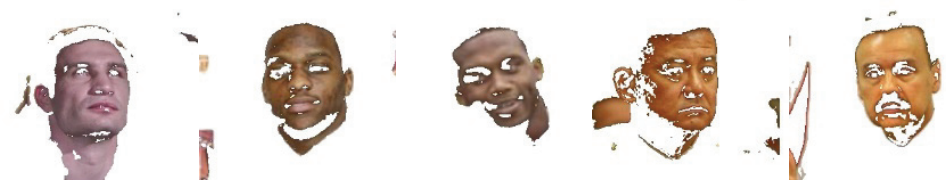

(l)

Fig. 4. Shows some of the results of the skin color segmentation process using different color models: (a) original images, (b) HSV, (c) IHLS, (d) RGB, (e) RGB\&YUV, (f) YCbCr, (g) YCgCr, (h) YIQ, (i) YIQ\&HSV, (j) YIQ\&YUV, (k) YUV, and (I) YUV \& RGB (proposed method)

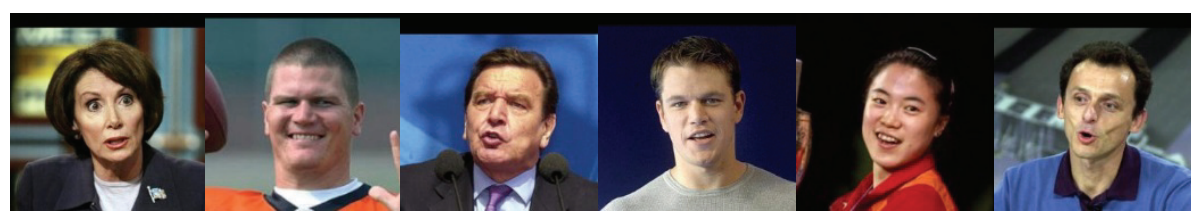

(a)
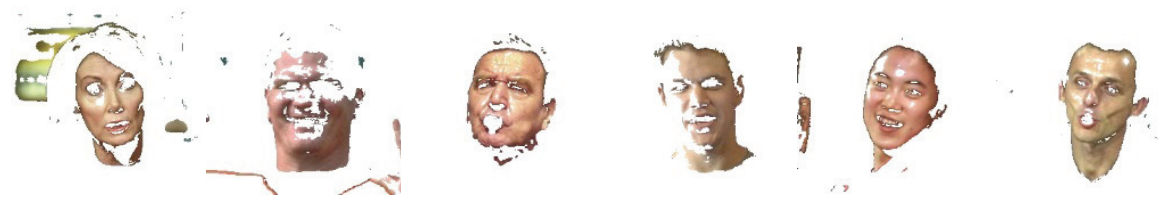

(b)
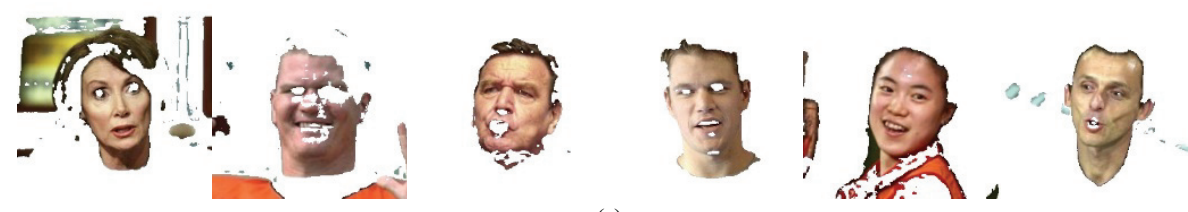

(c)
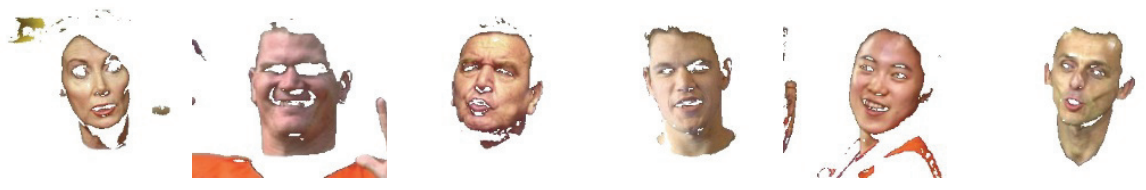

(d)
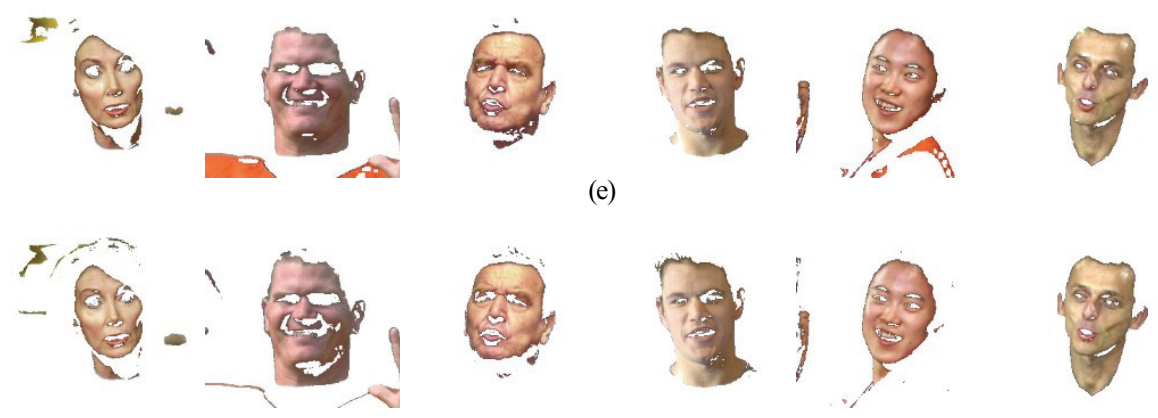

(f) 

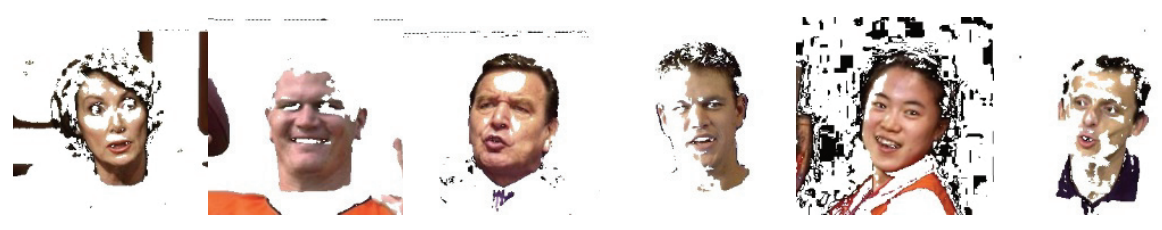

(g)
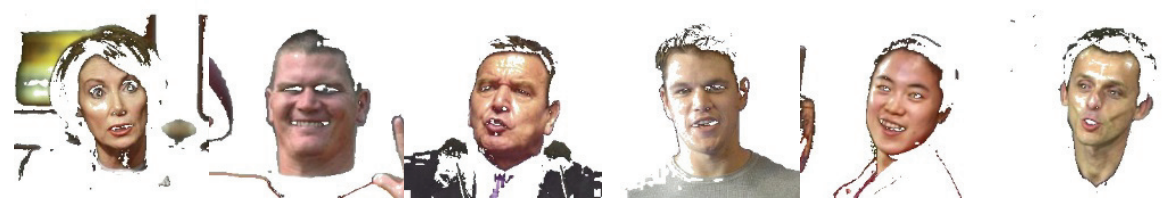

(h)
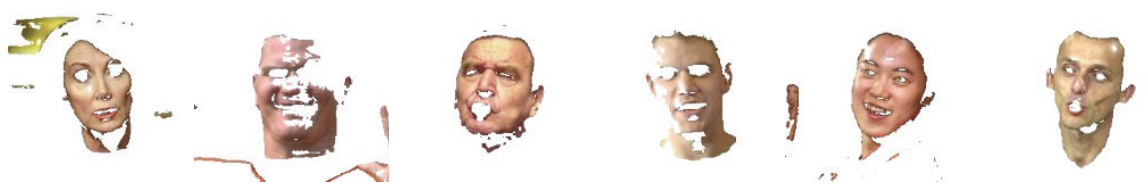

(i)
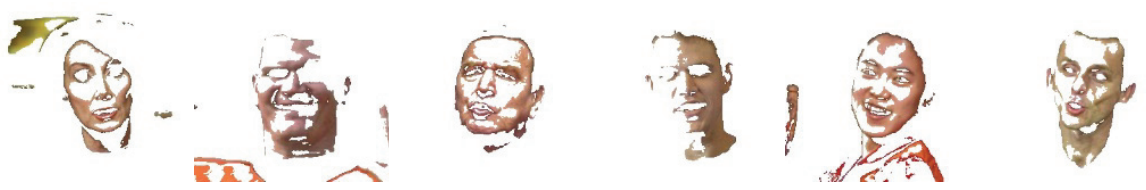

(j)
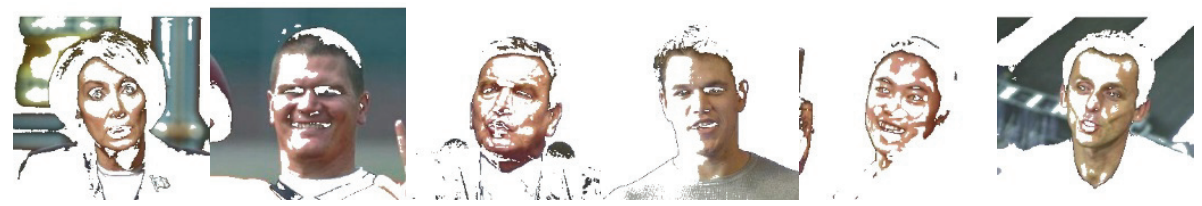

(k)
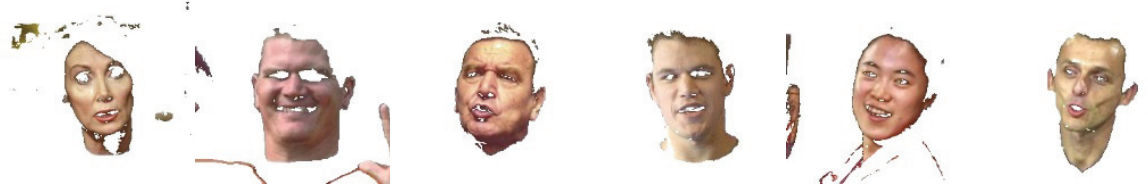

(1)

Fig. 5. Shows some of the results of the skin color segmentation process using different color models: (a) original images, (b) HSV, (c) IHLS, (d) RGB, (e) RGB\&YUV, (f) YCbCr, (g) YCgCr, (h) YIQ, (i) YIQ\&HSV, (j) YIQ\&YUV, (k) YUV, and (I) YUV \& RGB (proposed method)

\section{DISCUSSION}

Three different measures were used to evaluate the proposed method. The Detection Rate (DR) is defined as the number of pixels that are correctly detected as skin color pixels $(N s)$ over the 
total number of skin color pixels (Nf). The False Positive Rate (FPR) is defined as the number of non-skin pixels that are detected incorrectly as skin color ( $N f p)$ over the total number of nonskin color pixels (Nnf). The False Negative Rate (FNR) is defined as the number of skin color pixels that are detected incorrectly as non-skin color pixels (Nfn) over the total number of skin color pixels $(N f)$, as written by [25].

$$
\begin{gathered}
\text { Detection Rate }(\%)=\frac{\mathrm{Ns}}{\mathrm{Nf}} \times 100 \\
\text { False Positive Rate }(\%)=\frac{\mathrm{Nfp}}{\mathrm{Nnf}} \times 100 \\
\text { False Negative Rate }(\%)=\frac{\mathrm{Nfn}}{\mathrm{Nf}} \times 100
\end{gathered}
$$

These measures were used for achieving the comparison between our proposed method and the other methods. The results are shown in Table 1.

Table 1.Te performance of the proposed method and the other methods

\begin{tabular}{c|c|c|c}
\hline Method & DR & FPR & FNR \\
\hline HSV & 85.798 & 5.098 & 14.202 \\
\hline YIQ \& HSV & 85.845 & 4.834 & 14.155 \\
\hline YUV & 86.256 & 22.885 & 13.744 \\
\hline YIQ \& YUV & 86.706 & 8.923 & 13.294 \\
\hline YCgCr & 88.959 & 10.329 & 11.041 \\
\hline YCbCr & 89.647 & 7.580 & 10.353 \\
\hline IHLS & 90.868 & 11.760 & 9.132 \\
\hline YIQ & 91.759 & 18.868 & 8.241 \\
\hline RGB & 92.756 & 4.720 & 7.244 \\
\hline RGB \& YUV & 92.597 & 3.749 & 7.403 \\
\hline Our Proposed Method & 95.114 & 4.473 & 4.886 \\
\hline
\end{tabular}

From the results, we noticed that our proposed method has the highest Detection Rate (DR) value and the lowest False Positive Rate (FPR) and False Negative Rate (FNR) values.

\section{CONCLUSION}

Skin color segmentation is an important and challenging problem for many of the image processing and computer vision applications. To achieve better skin detection for images with complex backgrounds and different brightness, a new approach that is based on the RGB and YUV color spaces has been described. The results show that the proposed method can achieve a high skin detection rate compared to the other methods. In future work, other features, such as texture, will be used for more accurate detection in dealing with more complex backgrounds and colored hair that is similar to the skin color pixels. 


\section{REFERENCES}

[1] A. Cheddad, J. Condell, K. Curran, and P. McKevitt, "A new colour space for skin tone detection," in Proceedings of the 16th IEEE International Conference on Image Processing, Cairo, Egypt, 2009, pp. 497-500.

[2] G. Kukharev and A. Nowosielski, "Visitor identification: elaborating real time face recognition system," in Proceedings of the 12th International Conference in Central Europe on Computer Graphics, Visualization and Computer Vision, Plzen-Bory, Czech Republic, 2004, pp. 157-164.

[3] K. Sobottka and I. Pitas, "A novel method for automatic face segmentation, facial feature extraction and tracking," Signal Processing: Image Communication, vol. 12, no. 3, pp. 263-281, 1998.

[4] R. M. Jusoh, N. Hamzah, M. H. Marhaban, and N. M. A. Alias, "Skin detection based on thresholding in RGB and hue component," in IEEE Symposium on Industrial Electronics \& Applications, Penang, Malaysia, 2010, pp. 515-517.

[5] K. H. B. Ghazali, J. Ma, and R. Xiao, "An innovative face detection based on skin color segmentation," International Journal of Computer Applications, vol. 34, no. 2, pp. 6-10, 2011.

[6] A. S. Ghotkar and G. K. Kharate, "Hand segmentation techniques to hand gesture recognition for natural human computer interaction," International Journal of Human Computer Interaction, vol. 3, no. 1, pp. 15-25, 2012.

[7] B. N. Jagadesh, K. S. Rao, Ch. Satyanarayana, and G. V. S. RajKumar, "Skin colour segmentation using finite bivariate Pearsonian type-Iib mixture model and K-means," Signal \& Image Processing, vol. 3, no. 4, pp. 37-49, 2012.

[8] D. Chai and K. N. Ngan, "Face segmentation using skin-color map in videophone applications," IEEE Transactions on Circuits and Systems for Video Technology, vol. 9, no. 4, pp. 551-564, 1999.

[9] D. Marius, S. Pennathur, and K. Rose (2003). Face Detection Using Color Thresholding, and Eigenimage Template Matching [Online]. Available: http://scien.stanford.edu/pages/labsite/2003/ ee368/Project/slides/ee368group15.ppt

[10] J. J. de Dios and N. Garcia, "Fast face segmentation in component color space," in Proceedings of the International Conference on Image Processing, Singapore, 2004, pp. 191-194.

[11] A. H. K. Almohair, A. R. Ramli, A. M. Elsadig, and B. S. J. Hashim, "Skin detection in luminance images using threshold technique," International Journal of Imaging Science and Engineering, vol. 1, no. 1, pp. 32-35, 2007.

[12] M. S. Iraji and A. Yavari, "Skin color segmentation in fuzzy YCBCR color space with the Mamdani inference," American Journal of Scientific Research, vol. 2011, no. 7, pp. 131-137, 2011.

[13] C. Prema and D. Manimegalai, "A novel skin tone detection using hybrid approach by new color space," International Journal of Computer Applications, vol. 46, no. 7, pp. 15-19, 2012.

[14] J. Kovac, P. Peer, and F. Solina, "Human skin color clustering for face detection," in The IEEE Region 8 EUROCON 2003: Computer as a Tool, Ljubljana, Slovenia, 2003, pp. 144-148 vol.2.

[15] C. Prema and D. Manimegalai, "Survey on skin tone detection using color spaces," International Journal of Applied Information Systems, vol. 2, no. 2, pp. 18-26, 2012.

[16] A. Hanbury, "A 3D-polar coordinate colour representation well adapted to image analysis," in Image Analysis: 13th Scandinavian Conference, SCIA 2003 Halmstad, Sweden, June 29-July 2, 2003 Proceedings (Lecture Notes in Computer Science Vol. 2749), J. Bigun and T. Gustavsson, Eds., Heidelberg: Springer Berlin, 2003, pp. 804-811.

[17] R. Khan, Z. Khan, M. Aamir, and S. Q. Sattar, "Static filtered skin detection," International Journal of Computer Science Issues, vol. 9, no. 2, pp. 257-261, 2012.

[18] B. C. Ennehar, O. Brahim, and T. Hicham, "An appropriate color space to improve human skin detection," INFOCOMP Journal of Computer Science, vol. 9, no. 4, pp. 1-10, 2010.

[19] D. A. Lyon and N. Vincent, "Interactive embedded face recognition," Journal of Object Technology, vol. 8, no. 1, pp. 23-53, 2009.

[20] T. Abd El-Hafeez, "A new system for extracting and detecting skin color regions from PDF documents," International Journal on Computer Science and Engineering, vol. 2, no. 9, pp. 28382846, 2010.

[21] M. R. Tabassum, A. U. Gias, M. M. Kamal, S. Islam, H. M. Muctadir, M. Ibrahim, A. K. Shakir, A. 
Imran, S. Islam, M. G. Rabbani, S. M. Khaled, M. S. Islam, and Z. Begum, "Comparative study of statistical skin detection algorithms for sub-continental human images," Information Technology Journal, vol. 9, no. 4, pp. 811-817, 2010.

[22] F. H. Xiang and S. A. Suandi, "Fusion of multi color space for human skin region segmentation," International Journal of Information and Electronics Engineering, vol. 3, no. 2, pp. 172-174, 2013.

[23] J. J. de Dios and N. Garcia, "Face detection based on a new color space YCgCr," in Proceedings of the International Conference on Image Processing, Barcelona, Spain, 2003, pp. III-909-III-912.

[24] Labeled Faces in the Wild (Accessed 2012, August 25) [Online]. Available: http://viswww.cs.umass.edu/lfw/

[25] Y. Guoliang, L. Huan, Z. Li, and C. Yue, "Research on a skin color detection algorithm based on selfadaptive skin color model," in Proceedings of the International Conference on Communications and Intelligence Information Security, Nanning, China, 2010, pp. 266-270.

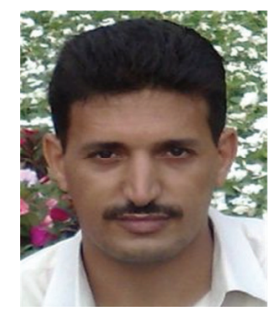

\section{Zaher Hamid Al-Tairi}

He received his B.Sc. in Computer Science from Thamar University in 2001 and M.Sc. in Multimedia System from Universiti Putra Malaysia, in 2008. He is a Computer Graphics doctoral student in faculty of Computer Science and Information Technology at University Putra Malaysia. His advisor is Dr. Rahmita Wirza. After college, he worked as a programmer at Central statistical Organisation and as a laboratory advisor at Thamar University. His research interests include Image Processing, Hand Interaction, Collision Detection, and Deformation Simulation.

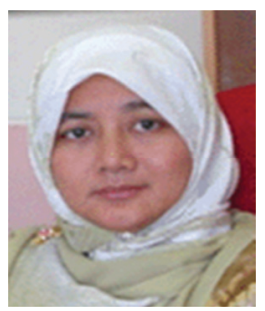

\section{Rahmita Wirza Rahmat}

She received the B.Sc. and M.Sc. degrees in Science Mathematics from University Science Malaysia, in 1989 and 1994, respectively. During 1989 to 1990 she works as research assistance in Department of Physics in University Science Malaysia, before working as tutor in Universiti Putra Malaysia. She received her $\mathrm{PhD}$ in Computer Assisted Engineering from University of Leeds, U.K. At this moment she is an Associate Professor working in Faculty of Computer Science and Information Technology as lecturer. Among her focus research area are Computer Graphics and Applications, Computer Assisted Surgery and Computational Geometry.

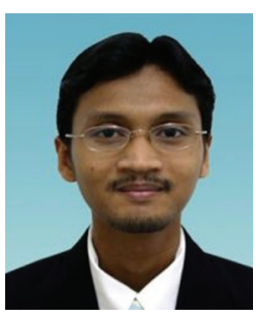

\section{Iqbal Saripan}

He received his B.Eng. degree in Electronics Engineering from Universiti Teknologi Malaysia in 2001 and PhD. Degree in Medical Imaging from University of Surrey, Guildford, U.K. in 2006. Currently, he is an Associate Professor at the Department of Computer and Communication Systems Engineering, Faculty of Engineering, Universiti Putra Malaysia. His research interests are in the area of Medical Imaging, Speech Processing, Artificial Intelligence, and Embedded System. Dr. Saripan is also a member of the IEEE and Institute of Physics UK. 


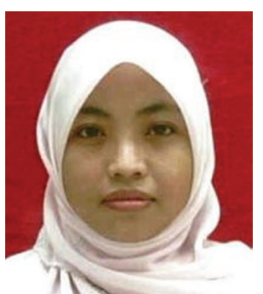

\section{Puteri Suhaiza Sulaiman}

She is currently a senior lecturer in the Department of Multimedia, Faculty of Computer Science and Information Technology at Universiti Putra Malaysia. She received her Ph.D. degree in Computer Graphic from Universiti Putra Malaysia, in 2010, M.S. in Computer Science from Universiti Teknologi Malaysia (UTM), in 2003, and Bac. in Computer Science from UTM, in 2000. Her research interests include Computer Graphics, Geographical Information Systems, Medical Visualization, Virtual Reality, and Computer Games. 